\title{
MEMS AND METAMATERIALS: A PERFECT MARRIAGE AT TERAHERTZ FREQUENCIES
}

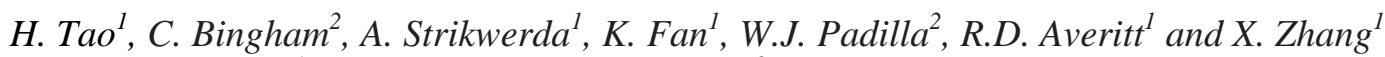 \\ ${ }^{1}$ Boston University, USA and ${ }^{2}$ Boston College, USA
}

\section{ABSTRACT}

This paper reports our recent progress on creating active structures and devices to enhance the ability to manipulate and detect far-infrared, or terahertz $(\mathrm{THz})$, radiation by combining electromagnetic metamaterials with MEMS technologies. We have designed, simulated, and fabricated state-of-the-art $\mathrm{THz}$ components which include a $\mathrm{THz}$ resonance switch, flexible $\mathrm{THz}$ metamaterials, and $3 \mathrm{D} \mathrm{THz}$ metamaterials. Preliminary results demonstrate the potential of MEMS-enhanced metamaterials for THz applications.

\section{INTRODUCTION}

The terahertz region of the electromagnetic spectrum extends from $100 \mathrm{GHz}$ to $10 \mathrm{THz}$ ( $1 \mathrm{THz}$ corresponds to a wavelength of 300 microns). This region, alternatively called the far-infrared, lies below visible and infrared wavelengths and above microwave wavelengths. This narrow portion of the electromagnetic spectrum is the least developed and therefore the least understood.

Motivating our effort to advance $\mathrm{THz}$ science and technology is the unique characteristics of $\mathrm{THz}$ radiation which includes transparency to materials such as cardboard, plastic, and styrofoam which are opaque at other wavelengths, and sensitivity to molecular signatures of gas phase and solid phase materials including biological agents and chemical explosives. While there have been laboratory-based demonstrations, further improvements in $\mathrm{THz}$ sources, components, and detectors are required for systems which are sufficiently compact and robust for real-world operation.

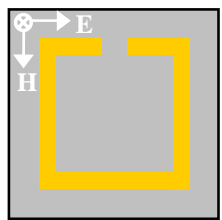

(a)

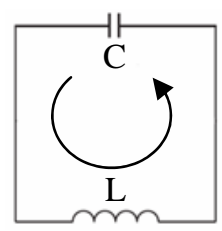

(d)

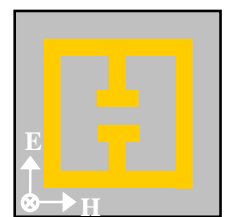

(b)

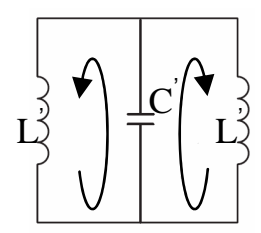

(e)

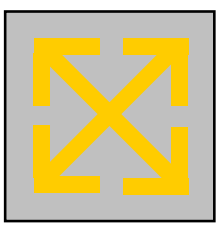

(c)

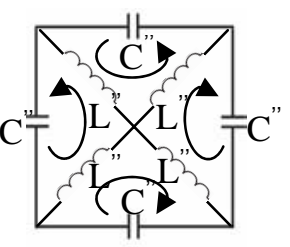

(f)
Figure 1: Some THz metamaterial split-ring resonators (SRRs) and equivalent circuits: a) \& d) Single-SRR, which shows bianisotropic responses, coupling to both electric and magnetic fields. b) \& e) Double-SRR, which couples strongly to a uniform electric field, and negligibly to a uniform magnetic field. c) \& f) Quad-SRR, which couples only to electric field, and is polarization non-sensitive due to high structure symmetry.

Recently, artificially structured electromagnetic materials have become an extremely active research area because of the possibility of creating materials which exhibit novel electromagnetic responses not available in natural materials, such as negative refractive index. Such electromagnetic composites, often called metamaterials, are sub-wavelength composites where the electromagnetic response originates from oscillating electrons in highly conducting metals such as gold or copper allowing for a design specific resonant response of the electrical permittivity or magnetic permeability, which are especially important for the technologically relevant THz frequency regime, as shown in Fig. 1.

\section{EXPERIMENTS}

In this paper, three different but correlated $\mathrm{THz}$ structures and components are developed through the intelligent design of metamaterial/MEMS composites, which show extreme power at microscale for sensing/imaging applications:

\section{Cantilever-based THz resonance switch}

An individual $\mathrm{THz}$ metamaterial resonance switch element consists of a split ring resonator (SRR) with a cantilever sitting above the gap, as shown in Fig. 2. Our device exhibits tunable electrically resonant response amplitude of nearly $50 \%$ at $0.75 \mathrm{THz}$ and operates by shorting the SRR gap with the bimaterial cantilevers. The modulation can be further improved by choosing high-Q SRRs and by optimizing the geometry of cantilevers.

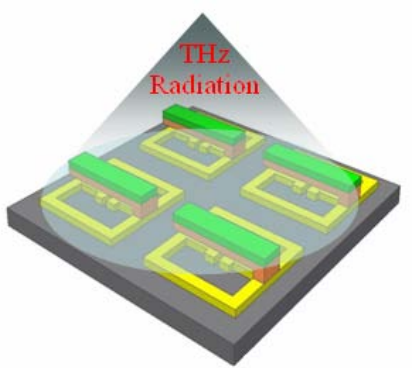

(a)

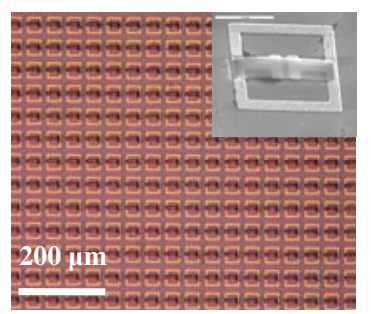

(c)

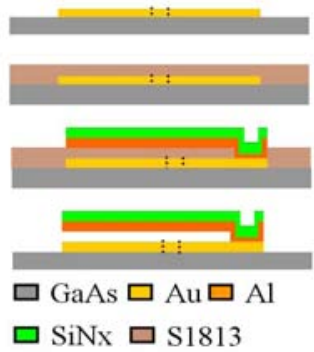

(b)

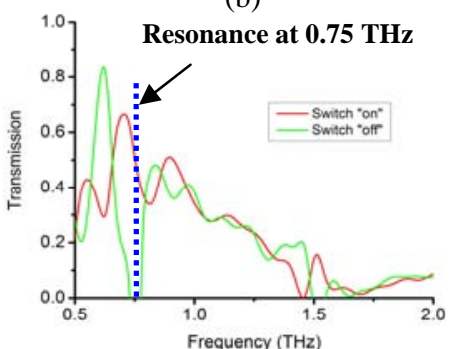

(d)
Figure 2: Cantilever-based $\mathrm{THz}$ resonance response switch: a) Planar periodic array exposed to normal incident radiation; b) Fabrication process flow; c) Photographs of the fabricated samples; d) Preliminary testing results showing a modulated resonance response of $\sim 50 \%$.

The switch was fabricated by using surface microfabrication process. A semi-insulating GaAs wafer was chosen because it is highly transmitting at $\mathrm{THz}$ frequencies; AZ5214e image reversal photoresist was patterned using direct laser writing with a Heidelberg $^{\mathrm{TM}}$ DWL 66 laser writer; 200 nm-thick Au/Ti was 
E-beam evaporated followed by lift-off with rinsing in Acetone for several minutes; $1.3 \mu \mathrm{m}$-thick photoresist of Shipley S1813 was spin-coated as the sacrificial layer; anchors were patterned by standard photolithography; a 200 nm-thick Al layer was E-beam evaporated, followed by sputtering of a $200 \mathrm{~nm}$-thick $\mathrm{SiN}_{\mathrm{x}}$ layer; cantilevers were patterned by standard photolithography, and etched by reactive ion etch of $\mathrm{SF}_{6} / \mathrm{He}$ and $\mathrm{Al}$ etchant respectively; isotropic release etching of photoresist was conducted by high density oxygen plasma asher.

Active controls of narrow band resonance response have been demonstrated before by optical pumping [1] and electric modulation [2]. This paper reports the first demonstration of the tunable metamaterial resonance response using the thermal-driven bimaterial cantilever switch realized by MEMS technologies.

\section{Flexible THz metamaterials}

A main charming of metamaterial is the potential of realization of "invisible cloak". The very first cloak device fabricated on PCB substrate was demonstrated at microwave frequency $(\sim 8.5 \mathrm{GHz})$, and named one of Science's top ten "Breakthroughs” of 2006 [3]. In this paper, we further pushed it into $\mathrm{THz}$ range by using MEMS technologies. Polyimide is chosen as the substrate material for $\mathrm{THz}$ "cloaking” because of: 1 ) its high transmission at $\mathrm{THz}$ frequencies; 2) its good mechanical flexibility - this is extremely important in the design of $\mathrm{THz}$ cloak, which composes multi-curved layers to provide gradually varying dielectric constants. In this paper, we demonstrate, for the first time, the advances for creating flexible metamaterials operating at the $\mathrm{THz}$ frequency range, as shown in Fig. 3.

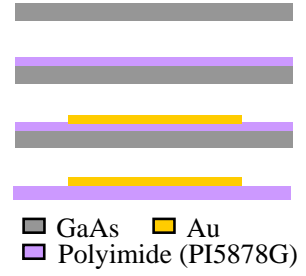

(a)

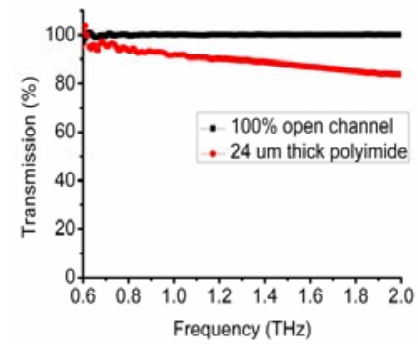

(c)

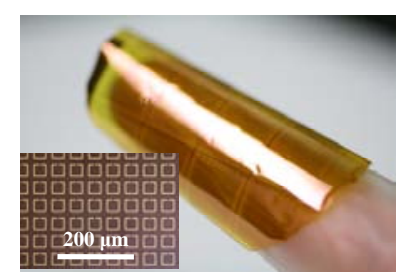

(b)

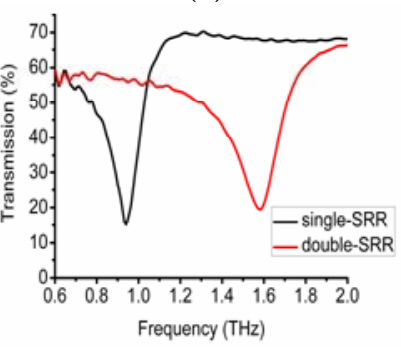

(d)
Figure 3: Flexible THz metamaterials: a) Fabrication process flow. (A 24 um-thick polyimide was spin-coated and cured on a silicon wafer; the SRR structures were patterned by using standard lift-off process, and then peeled off from the substrate.) b) A flexible "skin" applied to a finger. c) Transmission spectrum of the as-fabricated polyimide film. d) Measured resonance responses of single-SRR and double-SRR structures fabricated on flexible polyimide substrates.

\section{D THz metamaterials}

Most THz metamaterials are made in single planar layer and/or stack of multi planar layers. Although 3D structures were fabricated on silicon by using self-assembling techniques [4], the out-of-plane angle was relatively small and the silicon substrate was not transparent to $\mathrm{THz}$ radiation. In this paper, we demonstrate the first
3D THz metamaterials with large/controllable bending angles and high-filling factor using periodic pop-up structures suspended on low-stress $\mathrm{SiN}_{\mathrm{x}}$ films. The out-of-plane angle of $\sim 45^{\circ}$ was realized by bimaterial legs and further modified by rapid thermal annealing (RTA), as shown in Fig. 4.

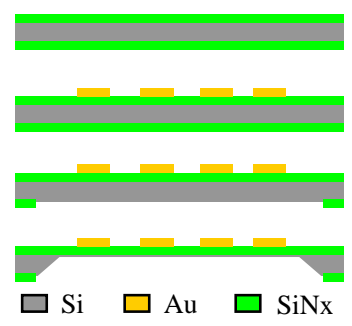

(a)

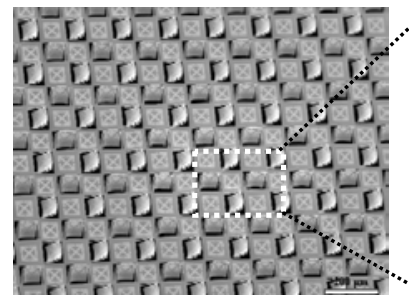

(c)

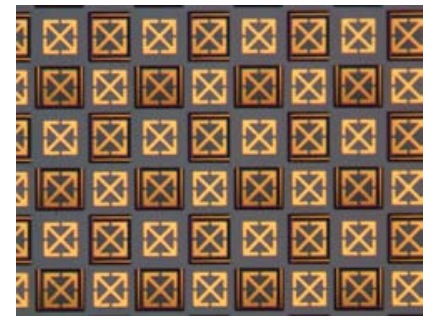

(b)

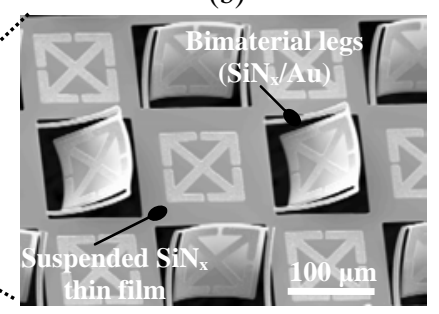

(d)
Figure 4: 3D THz metamaterials: a) Fabrication process flow: The SRR structures were patterned by using standard lift-off process on a silicon wafer with $400 \mathrm{~nm}$-thick $\mathrm{SiN}_{x}$ thin films deposited on both sides; the bimaterial legs were patterned by using lithography and RIE; open windows were patterned on the backside and then etched through in $\mathrm{KOH}$ solution to release the structure. b) Released structures before RTA. c) \& d) SEM photographs showing pop-up structures after $10 \mathrm{~min}$ RTA at $500^{\circ} \mathrm{C}$.

\section{CONCLUSION}

This paper introduces a compelling new research frontier in which functional $\mathrm{THz}$ metamaterial components were developed using MEMS technologies. It is believed that the results could set the stage for the marriage of metamaterials with MEMS, enabling technological advances that would otherwise be difficult to achieve.

\section{ACKNOWLEDGEMENTS}

This work is supported by DOD/Army Research Laboratory under grant W911NF-06-2-0040.

\section{REFERENCES}

[1] W.J. Padilla, A.J. Taylor, C. Highstrete, M. Lee, and R.D. Averitt, "Dynamical electric and magnetic metamaterial response at terahertz frequencies”, Physical Review Letters, 96, 107401 (2006).

[2] H.T. Chen, W.J. Padilla, J.M.O. Zide, A.C. Gossard, A.J. Taylor, and R.D. Averitt, "Active terahertz metamaterial devices”, Nature, 444, 597 (2006).

[3] D. Schurig, J.J. Mock, B.J. Justice, S.A. Cummer, J.B. Pendry, A.F. Starr, and D.R. Smith, "Metamaterial electromagnetic cloak at microwave frequencies”, Science, 314, 977 (2006).

[4] M.S. Islam and L. VJ, "Microfabrication of self-assembling elements for 3D negative-index materials", SPIE News room, DOI:10.117/2.1200612.0515, (2006). 